\title{
Inelastic neutron scattering studies on the odd-membered antiferromagnetic wheel $\mathrm{Cr}_{8} \mathrm{Ni}$
}

\author{
Michael L. Baker, ${ }^{1,2}$ Oliver Waldmann, ${ }^{3}$ Stergios Piligkos, ${ }^{4}$ Roland Bircher,${ }^{5}$ Olivier Cador, ${ }^{6}$ Stefano Carretta, ${ }^{7}$ \\ David Collison, ${ }^{2}$ Felix Fernandez-Alonso, ${ }^{8}$ Eric J. L. McInnes, ${ }^{2}$ Hannu Mutka, ${ }^{1}$ Andrew Podlesnyak, ${ }^{9}$ Floriana Tuna,,${ }^{2,10}$ \\ Stefan Ochsenbein, ${ }^{5}$ Roberta Sessoli, ${ }^{6}$ Andreas Sieber, ${ }^{5}$ Grigore A. Timco, ${ }^{2}$ Høgni Weihe ${ }^{4}$ Hans U. Güdel, ${ }^{5}$ and \\ Richard E. P. Winpenny ${ }^{2,11}$ \\ ${ }_{1}^{1}$ Institut Laue-Langevin, BP 156, 6 rue Jules Horowitz, 38042 Grenoble Cedex 9, France \\ ${ }^{2}$ School of Chemistry, The University of Manchester, Oxford Road, Manchester, M13 9PL, United Kingdom \\ ${ }^{3}$ Physikalisches Institut, Universität Freiburg, D-79104 Freiburg, Germany \\ ${ }^{4}$ Department of Chemistry, University of Copenhagen Universitetsparken 5, 2100 Copenhagen, Denmark \\ ${ }^{5}$ Department of Chemistry and Biochemistry, University of Bern, Freiestrasse 3, 3000 Bern 9, Switzerland \\ ${ }^{6}$ Laboratorio di Magnetismo Molecolare Dipartimento di Chimica and INSTM, Universitá degli Studi di Firenze, 50019 Sesto Fiorentino, Italy \\ ${ }^{7}$ Dipartimento di Fisica, Universitá di Parma and CNISM, I-43124 Parma, Italy \\ ${ }^{8}$ ISIS Facility, Rutherford Appleton Laboratory, Chilton, Didcot, Oxfordshire OX11 OQX, United Kingdom* \\ ${ }^{9}$ Quantum Condensed Matter Division, Oak Ridge National Laboratory, Oak Ridge, Tennessee 37831, USA \\ ${ }^{10}$ The EPSRC National UK EPR Facility, Photon Science Institute, University of Manchester, Oxford Road, M13 9PL, United Kingdom \\ ${ }^{11}$ Photon Science Institute, University of Manchester, Oxford Road, Manchester M13 9PL, United Kingdom
}

(Received 11 May 2012; published 3 August 2012)

\begin{abstract}
$\left[\left({ }^{\mathrm{i}} \mathrm{C}_{3} \mathrm{H}_{7}\right)_{2} \mathrm{NH}_{2}\right]\left[\mathrm{Cr}_{8} \mathrm{NiF}_{9}\left(\mathrm{O}_{2} \mathrm{CCMe}\right)_{18}\right]$, or $\mathrm{Cr}_{8} \mathrm{Ni}$, is a prominent example of an odd-membered antiferromagnetic "wheel." A detailed characterization of the magnetic properties of $\mathrm{Cr}_{8} \mathrm{Ni}$ has been conducted. Inelastic neutron scattering (INS) is used to investigate the energy and momentum transfer dependence of the low-lying spin excitations, including excited states inaccessible by other experimental techniques. The richness of the INS data, in conjunction with microscopic spin Hamiltonian simulations, enables an accurate characterization of the magnetic properties of $\mathrm{Cr}_{8} \mathrm{Ni}$. Nearest-neighbor exchange constants of $J_{\mathrm{CrCr}}=1.31 \mathrm{meV}$ and $J_{\mathrm{CrNi}}=3.22 \mathrm{meV}$ are determined, and clear evidence of axial single-ion anisotropy is found. The parameters determined by INS are shown to fit magnetic susceptibility. The spectroscopic identification of several successive $S=1$ excited total spin states and lowest spin band excitations show that the rotational band picture, valid for bipartite AFM wheels, breaks down for this odd-numbered wheel. The exchange constants determined here differ from previous efforts based on bulk measurements, and possible reasons are discussed. The large $J_{\mathrm{CrNi}} / J_{\mathrm{CrCr}}$ ratio in $\mathrm{Cr}_{8} \mathrm{Ni}$ puts this wheel into a regime with strong quantum fluctuations in which the ground state can be described with a valence bond solid state picture.
\end{abstract}

DOI: 10.1103/PhysRevB.86.064405

PACS number(s): 75.50.Xx, 75.40.Gb, 75.10.Jm, 78.70.Nx

\section{INTRODUCTION}

Molecular nanomagnets provide a means to investigate the physical properties of spin clusters of finite size, which contain typically a dozen or so magnetic transition metal ions. Each cluster is magnetically isolated from its neighbors by bulky organic ligands, providing some of the best examples of zero-dimensional magnetic systems. Varying the magnetic exchange pathways and the number and types of the magnetic metal ions in the clusters can markedly affect the physical properties, and the molecular nanomagnets provide a rich playground for studying the magnetism at the nanoscale. ${ }^{1-6}$

In, for instance, antiferromagnetic (AFM) wheels with an even number of exchange-coupled magnetic ions the low-lying spin excitations can be described by a simplified Hamiltonian model, ${ }^{7,8}$ which reduces the system to two magnetic sublattices. The spins within each sublattice group remain parallel, where the total spin on sublattice $\mathrm{A}$ is equivalent to that on sublattice B, and the total spin ground state is zero. Such bipartite systems ${ }^{9}$ can be described remarkably well by an effective Hamiltonian that couples the two effective sublattice spins only. Inelastic neutron scattering (INS) has provided the means to confirm this model on a variety of even AFM wheels. ${ }^{10-13}$ Additionally, AFM wheels have shown interesting quantum phenomena, such as quantum tunneling oscillations in the homonuclear wheel $\mathrm{Fe}_{18}$ or coherent oscillation of the total quantum spin at avoided level crossings in applied magnetic fields in the heterometallic AFM wheel $\mathrm{Cr}_{7} \mathrm{Ni}^{13,14}$

As distinguished from the even wheels, the topology of odd-membered AFM wheels is of separate interest because of the possibility of boundary-induced frustration effects. Spin frustration can occur when competing AFM couplings between spin moments causes the interactions to be not satisfied simultaneously. An isolated AFM spin-half triangle represents the simplest example, where the ground state is fourfold degenerate, forming two total spin $S=1 / 2$ multiplets. Spectroscopic studies on such spin triangles have shown that usually it is energetically favorable for the ground-state degeneracy to be (partially) removed via structural distortions ${ }^{15-17}$ or antisymmetric exchange between the local spins. ${ }^{18}$ Spin triangles are the "building blocks" in most extended magnetic systems displaying spin frustration effects, ${ }^{19}$ and some of the underlying principles imposed by magnetic frustration are revealed by their molecular based counterparts. ${ }^{1,20,21}$ As opposed to the half-spin triangles, or triangles with an odd number of magnetic electrons in general, in which spin frustration reveals itself at the quantum level through the degeneracy in the ground state, spin triangles with an even number of electrons exhibit a $S=0$ ground state and the notion 


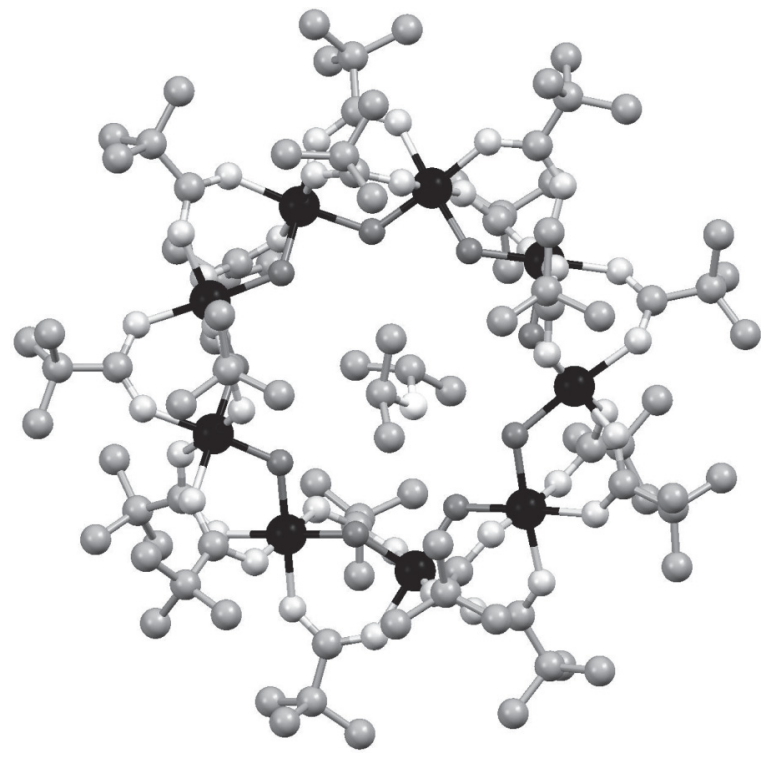

FIG. 1. The Structure of the $\mathrm{Cr}_{8} \mathrm{Ni}$. Cr and $\mathrm{Ni}$ ions are represented by large black spheres, where the $\mathrm{Ni}$ position is disordered over the nine possible metal sites, $\mathrm{O}$ are light gray spheres, $\mathrm{C}$ gray spheres, $\mathrm{F}$ dark gray spheres, and $\mathrm{N}$ in the center of the wheel is represented by a white sphere. $\mathrm{H}$ atoms are omitted for clarity.

of frustration becomes less obvious despite the competing AFM interactions in them. In larger lattices, or extended lattices, with an $S=0$ ground-state spin frustration may, however, be signaled by so-called low-lying singlets. ${ }^{19}$

Beyond spin triangles, one molecular shape of particular interest is the AFM wheel with an odd number of exchangecoupled metal centers. The title compound, the nona-nuclear hetero-metallic wheel $\left[\left({ }^{\mathrm{i}} \mathrm{C}_{3} \mathrm{H}_{7}\right)_{2} \mathrm{NH}_{2}\right]\left[\mathrm{Cr}_{8} \mathrm{NiF}_{9}\left(\mathrm{O}_{2} \mathrm{CCMe}\right)_{18}\right]$, or $\mathrm{Cr}_{8} \mathrm{Ni}$ in short, has been one of the first reported odd AFM wheels. It consists of eight $\mathrm{Cr}$ (III) (spin 3/2) and one $\mathrm{Ni}(\mathrm{II})$ (spin 1) arranged into a ring structure, see Fig. 1. Examples of odd AFM wheels are extremely rare as compared to their even membered relatives. Currently, reported examples of odd-membered macrocyclic systems with significant AFM interactions beyond triangles include a nona-nuclear ferric wheel and a heptagonal oxovanadium wheel only. ${ }^{22,23}$ The synthesis of $\mathrm{Cr}_{8} \mathrm{Ni}$ was first communicated in Ref. 24, and its magnetic properties have since then received much attention $^{25-28}$ as the spin configuration in the classical ground state was identified to be characterized as a magnetic Möbius strip. $^{24}$

Owing to the even number of magnetic electrons in $\mathrm{Cr}_{8} \mathrm{Ni}$, the exchange spectrum is characterized by spin multiplets with integer total spin quantum number $S$, and the ground state was determined to belong to $S=0$. In this regard, the situation concerning frustration is similar to that in integer-spin triangles. However, in contrast to the triangles and the related lattices, the frustration in odd AFM wheels is not due to a direct competition of two exchange bonds but the incompatibility of the closed boundary conditions with a bipartite sublattice structure. It also cannot be captured by a tripartite sublattice structure, which allowed to successfully describe trianglebased frustrated magnetic molecules such as the Keplerate
$\mathrm{Fe}_{30} \cdot{ }^{29}$ The lack of partitedness at any spatial range puts the odd AFM wheels into an interesting class of materials by itself.

The bi- and tripartite sublattice structure in molecules such as the even AFM wheels or the $\mathrm{Fe}_{30}$ molecule allowed to devise effective spin Hamiltonians, which provided much insight into the physics in these systems as described before. Obviously, such approaches cannot be applied to the odd AFM wheels, and our understanding of the energies and wave functions in them, beyond the insight obtained through numerical calculations, ${ }^{30,31}$ is still poor. In a recent theoretical study, it was proposed that the ground state in $\mathrm{Cr}_{8} \mathrm{Ni}$ can be characterized as a valence-bond solid (VBS). ${ }^{28,32}$ Valencebond theory is a convenient framework in the investigation of ground state properties of various extended AFM spin systems with disordered ground states ${ }^{33,34}$ and was applied to generic frustrated magnets such as pyrochlore ${ }^{35}$ and square lattice $^{36}$ extended systems. Within the valence-bond picture, the presence of the $\mathrm{Ni}(\mathrm{II})$ ion in $\mathrm{Cr}_{8} \mathrm{Ni}$ forces the ground state into a VBS if the exchange between the $\mathrm{Cr}$ ions is weak as compared to those involving the $\mathrm{Ni}$ ion. In this state, single valence bonds are attached to the $\mathrm{Ni}$ (II) atom and double valence bonds are then fixed to specific positions around the wheel, inducing a alternating coupling pattern around the wheel. $^{28}$

Several experiments on $\mathrm{Cr}_{8} \mathrm{Ni}$ have previously been performed investigating bulk magnetization, specific heat, and electron spin resonance. ${ }^{24,25}$ They allowed characterizing the lowest-lying band of total spin states. The structure of the excitation spectrum and the presence of zero-field splitting (ZFS) remains, however, somewhat unknown. A detailed study of the low-lying spin states in $\mathrm{Cr}_{8} \mathrm{Ni}$ is hence of upmost importance, and we here report an inelastic neutron scattering (INS) study. INS investigations on $\mathrm{Cr}_{8} \mathrm{Ni}$ offer a direct probe to the energy and momentum transfer of low-lying states, including those inaccessible to previously reported techniques.

\section{EXPERIMENTS}

The INS experiments on $\mathrm{Cr}_{8} \mathrm{Ni}$ were performed on several neutron scattering instruments, namely, the direct time-offlight spectrometer IN5 at Institute Laue-Langevin, the direct time-of-flight spectrometer FOCUS at the Swiss spallation neutron source SINQ, Paul Scherrer Institute, and the inverse geometry time-of-flight spectrometer IRIS at ISIS, Science and Technology Facilities Council Rutherford Appleton Laboratory. A crystalline powder sample of $\mathrm{Cr}_{8} \mathrm{Ni}$ was prepared for these experiments to the specification outlined in Ref. 37. The sample was sealed inside an aluminum cylinder. Measurements were performed at various temperatures within a range from $1.5-18 \mathrm{~K}$. IN5 data were measured with several chopper settings, where different speeds and ratios were used to select the optimum resolution, energy and momentum transfer ranges. IN5 measurements at $4.0 \AA$ were made with two chopper settings; $2.0 \mathrm{~K}$ data were measured with a $17500 \mathrm{rpm}$ chopper setting, 6.0 and $14.5 \mathrm{~K}$ measurements were performed with a $15000 \mathrm{rpm}$ setting. The higher chopper speed provides an instrumental energy resolution at the elastic line of full width half maximum (FWHM) of $0.134 \mathrm{meV}$, $10 \mu \mathrm{eV}$ higher than the $15000 \mathrm{rpm}$ setting, enabling greater separation of the first cold excitation from the elastic scattered 
intensity. A shorter wavelength of $3.6 \AA$ probed higher-energy transfers with an instrumental energy resolution at the elastic line of FWHM of $0.178 \mathrm{meV}$. Measurements using the FOCUS spectrometer were performed with a $3.5 \AA$ incident neutron wavelength providing a FWHM of $0.29 \mathrm{meV}$ at the elastic line. IRIS measurements were performed with the PG002 analyzer crystal setting; the instrumental resolution is FWHM $17.5 \mu \mathrm{eV}$ at zero energy transfer. All spectra were corrected for detector efficiencies by vanadium normalization. IN5 data measured with $4.0 \AA$ incident neutron wavelength are sample environment and sample holder subtracted. In addition to scattering angle integrated energy spectra, the momentum transfer $(Q)$ dependence was investigated by integration over energy regions corresponding to the maxima of a magnetic exchange transition. Magnetic scattering intensity was strong enough to express scattering in $Q$ and energy transfer, $S(Q, \omega)$, providing a rich overview of the magnetic bands. Bulk susceptibility measurements were performed in the temperature range of 1.8-300 K using a Quantum Design MPMS-XL SQUID magnetometer equipped with a $7 \mathrm{~T}$ magnet. Diamagnetic corrections were estimated using Pascal's constants and magnetic measurements were corrected for sample holder contributions. In-house software was used to simulate the experimental INS spectra and susceptibility.

\section{THE HAMILTONIAN MODEL}

The properties of AFM wheels can be described by a quantum spin Hamiltonian, which for $\mathrm{Cr}_{8} \mathrm{Ni}$ is expressed as follows:

$$
\begin{aligned}
\hat{H}= & J_{\mathrm{CrCr}} \sum_{i=1}^{7} \hat{\mathbf{s}}_{i} \cdot \hat{\mathbf{s}}_{i+1}+J_{\mathrm{CrNi}}\left(\hat{\mathbf{s}}_{1} \cdot \hat{\mathbf{s}}_{0}+\hat{\mathbf{s}}_{8} \cdot \hat{\mathbf{s}}_{0}\right) \\
& +D_{\mathrm{Cr}} \sum_{i=1}^{8}\left[\hat{s}_{z, i}^{2}-\frac{1}{3} s_{i}\left(s_{i}+1\right)\right] \\
& +D_{\mathrm{Ni}}\left[\hat{s}_{z, 0}^{2}-\frac{1}{3} s_{0}\left(s_{0}+1\right)\right] \\
& -g_{\mathrm{Cr}} \mu_{B} \sum_{i=1}^{8} \mathbf{H} \cdot \hat{\mathbf{s}}_{i}-g_{\mathrm{Ni}} \mu_{B} \mathbf{H} \cdot \hat{\mathbf{s}}_{0},
\end{aligned}
$$

where $\hat{\mathbf{s}}_{1}$ to $\hat{\mathbf{s}}_{8}$ represent the $\mathrm{Cr}(\mathrm{III})$ spins of length $s=$ $3 / 2$ and $\hat{\mathbf{s}}_{0}$ the $\mathrm{Ni}(\mathrm{II})$ spin of length $s_{0}=1$. The first two terms in the Hamiltonian operator (1) describe the dominant isotropic Heisenberg-Dirac-van Vleck AFM exchange couplings between nearest-neighbor metal ions. Exchange couplings between $\mathrm{Cr}$ ions are approximated as equivalent, and the two $\mathrm{Cr}$ to $\mathrm{Ni}$ exchanges are distinct from $\mathrm{Cr}-\mathrm{Cr}$ exchange, while equivalent to each other. The third and forth terms in the Hamiltonian operator (1) describe single-ion axial anisotropy for the $\mathrm{Cr}$ (III) and $\mathrm{Ni}(\mathrm{II})$ ions, where $D_{\mathrm{Cr}}$ and $D_{\mathrm{Ni}}$ are the anisotropy parameters. The anisotropic part of the Hamiltonian is simplified; the axes of the anisotropy for each metal ion site are collinear with each other and assumed to run with principal axis perpendicular to the plane of the wheel. Anisotropic dipolar interactions between unpaired elections are approximated to be included within the single-ion terms.
Nonaxial terms are not included; the axial zero-field-splitting (ZFS) term is the most appropriate.

Exact diagonalization of the total Hilbert space corresponding to the full $\mathrm{Cr}_{8} \mathrm{Ni}$ Hamiltonian matrix is computationally demanding. We used a variety of numerical methods to calculate the energies and wave function, and wherefrom the observables such as the INS spectra for which the formulaes given in Refs. 38-40 were used. Depending on the situation, symmetry-adapted irreducible tensor operator based full exact diagonalization $^{41}$ as well as sparse matrix exact diagonalization techniques were used. Specifically, for the simulation of experimental data, an iterative process was used, which utilized the sparsity of the full Hamiltonian matrix to reduce the full matrix to a subspace including the experimentally probed lowest-lying states. This method, relying on the Davidson algorithm, ${ }^{42}$ has been used previously in Ref. 43, and more recently to successfully simulate EPR, MCD, and INS spectra of even membered heterometallic wheels. ${ }^{44-46}$ Eigenvalues of the states probed in the INS experiment are included, along with higher-energy states within a computationally manageable subspace matrix. The number of states included in the subspace is tested by simulation of INS spectra for increasing subspace size until contributions from higher-lying spin states is negligible relative to experimental error of the measured spectra. We found a subspace including the first $70 m_{s}$ eigenstates to be sufficient. The reduced matrix makes calculations reasonable in terms of computational demand, while including all the eigenstates accessed in the INS experiment, including higher states connected to probed states by off diagonal anisotropy terms.

\section{EXPERIMENTAL RESULTS AND ANALYSIS}

Figure 2 presents neutron-energy loss spectra recorded on the FOCUS spectrometer at temperatures of 1.5, 6.0, and $18 \mathrm{~K}$. Significant incoherent scattering from the ligand atoms with large neutron cross sections is observed. On top of the incoherent scattering background three inelastic features

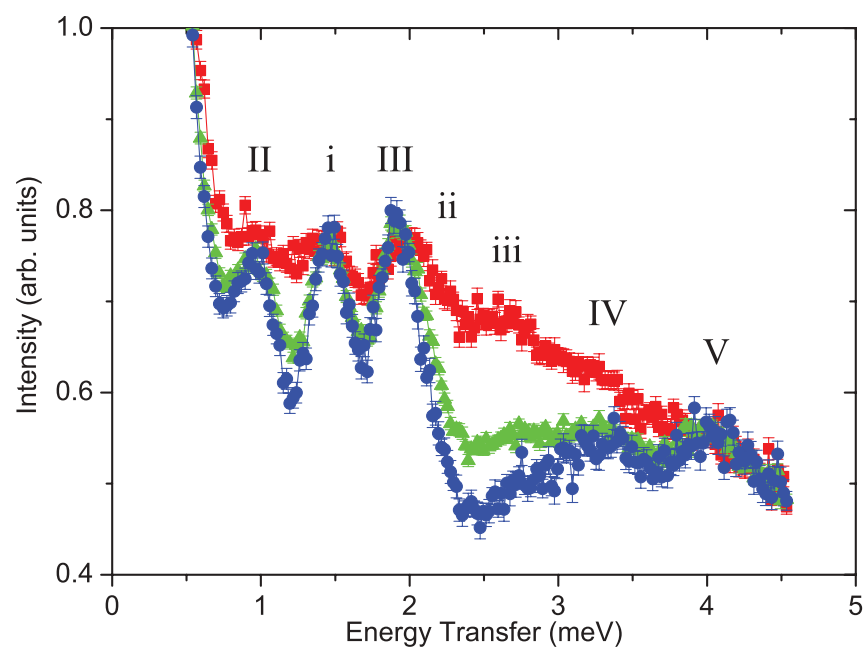

FIG. 2. (Color online) INS intensity as a function of energy transfer measured on FOCUS with a $3.5-\AA$ incident neutron wavelength at $2 \mathrm{~K}$ (blue circles), $6 \mathrm{~K}$ (green triangles), and $18 \mathrm{~K}$ (red squares). Transition labels are discussed in the text. 


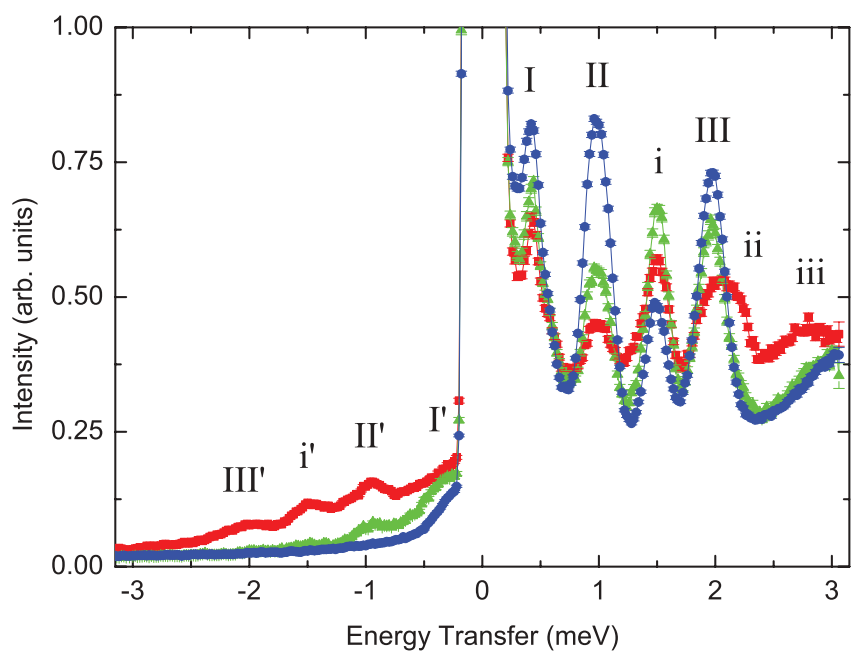

FIG. 3. (Color online) INS intensity as a function of energy transfer measured on IN5 with a 4.0- $\AA$ incident neutron wavelength at $2 \mathrm{~K}$ (blue circles), $6 \mathrm{~K}$ (green triangles), and $14.5 \mathrm{~K}$ (red squares). Transition labels are discussed in the text.

centered at 1.0 (peak II), 1.5 (peak i), and $2.0 \mathrm{meV}$ (peak III) dominate the spectra at 1.5 and $6 \mathrm{~K}$, and less well resolved features at approximately 3.4 (IV) and $4.0 \mathrm{meV}$ (VI) are observed. At $18 \mathrm{~K}$, nonmagnetic phononic scattering contributions dominate the spectrum, however broad, weak maxima at approximately 2.2 (peak ii) and $2.6 \mathrm{meV}$ (peak iii) suggests hot transitions emanating from excited states.

The measurements performed on the IN5 spectrometer revealed detailed temperature and $Q$ dependent information. The temperature-dependent, $Q$ integrated neutron spectra at 2.0, 6.0, and $14.5 \mathrm{~K}$ are presented in Fig. 3. The transitions I to III and $i$ to iii detected in the FOCUS data are clearly observed with good resolution, and the transfer energies are determined to 0.42 (I), 0.98 (II), and $1.94 \mathrm{meV}$ (III) and 1.50 (i), 2.13 (ii), and $2.85 \mathrm{meV}$ (iii). From the temperature dependence, features I, II, and III are clearly determined as cold magnetic transitions from the ground state and i, ii, and iii as hot magnetic transitions. On the anti-Stokes sides of the spectra (negative in energy transfer) the associated down-scattering transitions, I', II', III', and i' are observed at 6 and $14.5 \mathrm{~K}$, providing additional confirmation that the transitions are of magnetic scattering origin. On the anti-Stokes side, instrument energy resolution is lower as compared to the Stokes side and hence

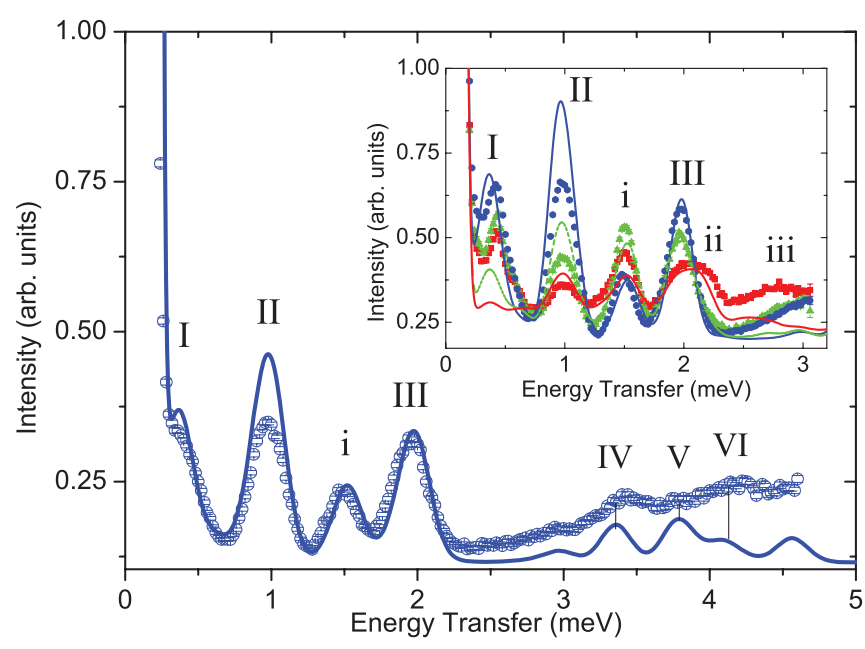

FIG. 5. (Color online) Neutron-energy loss intensity as a function of energy transfer measured on IN5 with $3.6-\AA$ incident neutron wavelength at $2.5 \mathrm{~K}$ (blue circles). The blue solid line shows theoretical simulated results. The insert shows $4.0 \AA$ incident neutron wavelength IN5 spectra at 2 (blue circles), 6 (green triangles), and $14.5 \mathrm{~K}$ (red squares) with simulated curves represented by solid lines. Simulations are based on (1) with exchange coupling constants of $J_{\mathrm{CrCr}}=1.32 \mathrm{meV}$ and $J_{\mathrm{CrNi}}=3.22 \mathrm{meV}$ and axial single-ion anisotropy $D_{\mathrm{Cr}}=-0.036 \mathrm{meV}$ and $D_{\mathrm{Ni}}=+1.25 \mathrm{meV}$.

the data on the Stokes side were used for determining energy positions. The neutron scattering intensity as a function of $Q$ and energy transfer $S(Q, \omega)$ is particularly well defined for $\mathrm{Cr}_{8} \mathrm{Ni}$, providing a detailed view on the magnetic excitations, as is shown for the 2.0-K 4.0-Å measurement in Fig. 4(a). The magnetic nature of the excitations are clearly identified by their oscillatory $Q$ dependence of the scattering intensity. The four transitions I, II, i, and III all show similar $Q$ dependencies, with a broad maximum between 1.25 and $1.5 \AA^{-1}$.

Measurements at IN5 with a shorter wavelength of $3.6 \AA$ enabled access to larger energy transfer, forfeiting energy resolution relative to the $4.0 \AA$ spectra. At $2.5 \mathrm{~K}$, evidence of cold transitions at 3.4 (IV) and $4.2 \mathrm{meV}$ (VI), and possibly at $3.8 \mathrm{meV}(\mathrm{V})$, are observed (see Fig. 5). The magnetic features lie on top of a significant nonmagnetic scattering background, which increases in a nonlinear fashion with increasing energy transfer.

The INS measurements performed on the spectrometer IRIS allowed probing the lowest-lying spin excitations in $\mathrm{Cr}_{8} \mathrm{Ni}$
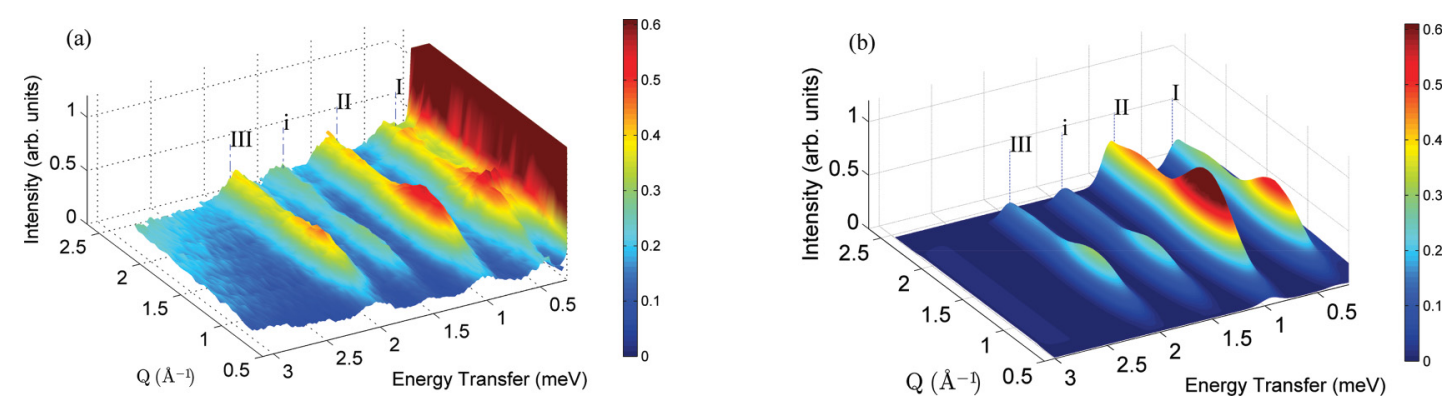

FIG. 4. (Color) (a) INS as a function of wave vector and energy transfer measured on IN5 with a 4.0- $\AA$ incident wavelength setting at $2 \mathrm{~K}$. (b) Simulation based on equation (1) using exchange coupling constants $J_{\mathrm{CrCr}}=1.31 \mathrm{meV}$ and $J_{\mathrm{CrNi}}=3.22 \mathrm{meV}$ and axial single-ion anisotropy $D_{\mathrm{Cr}}=-0.036 \mathrm{meV}$ and $D_{\mathrm{Ni}}=+1.25 \mathrm{meV}$. 


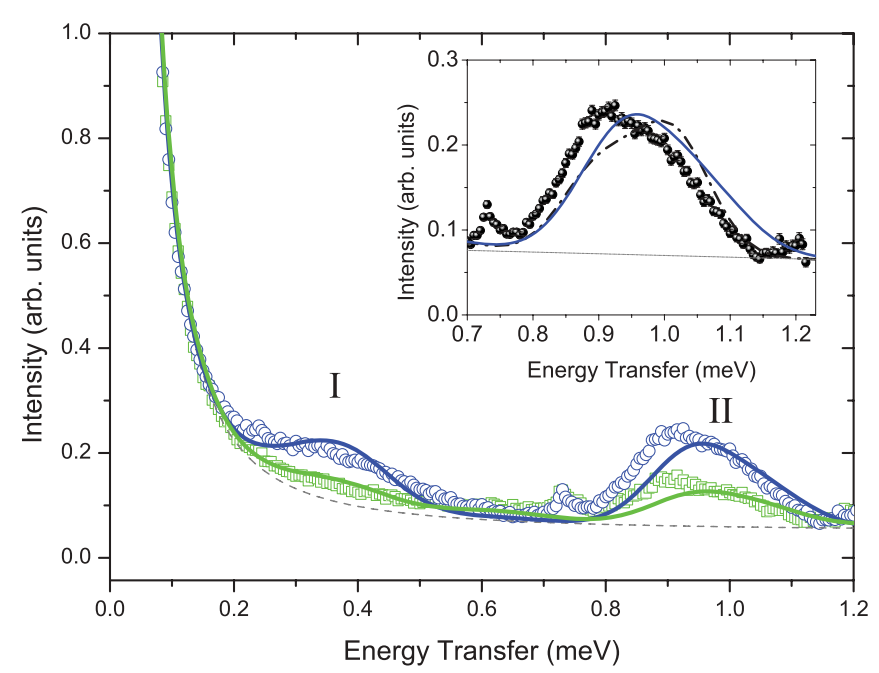

FIG. 6. (Color online) INS intensity as a function of energy transfer measured on IRIS. The blue open circles and green open squares show 1.5 and $6 \mathrm{~K}$ spectra, respectively. Solid lines show simulated spectra based on (1) with exchange coupling constants of $J_{\mathrm{CrCr}}=1.32 \mathrm{meV}$ and $J_{\mathrm{CrNi}}=3.22 \mathrm{meV}$, and axial single-ion anisotropy $D_{\mathrm{Cr}}=-0.036 \mathrm{meV}$ and $D_{\mathrm{Ni}}=+1.25 \mathrm{meV}$. The broken line represents the elastic line and background contributions of the simulated spectra. The insert shows the $1.5 \mathrm{~K}$ spectrum near feature II in more detail (black circles) and the solid blue line represents the simulation using $D_{\mathrm{Ni}}=+1.25 \mathrm{meV}$ and the dash-dotted black line that for $D_{\mathrm{Ni}}=-0.43 \mathrm{meV}$.

with high resolution. In the $1.5-\mathrm{K}$ spectrum, transitions I and II are clearly observed (see Fig. 6). However, both transition I and II have a FWHM, which is considerably larger than the instrumental resolution at these energy transfers, namely, 23 and $28 \mu \mathrm{eV}$ at 0.4 and $0.9 \mathrm{meV}$, respectively. The asymmetry of feature II suggests that it consists of two lines of different intensity with an energy difference of approximately $0.1 \mathrm{meV}$, which are not fully resolved in the experiment (see also inset to Fig. 6).

On a qualitative level the experimental INS data may be analyzed following two different scenarios, depending on the interpretation of the nature of peaks I and II. In view of the typical ZFS of the first excited $S=1$ spin multiplet of several $0.1 \mathrm{meV}$ observed in a number of even AFM wheels, ${ }^{10-13}$ features I and II could be associated to the transitions from the $S=0$ ground state into the first excited $S=1$ multiplet, which is split by anisotropy into its $M=0$ and $M= \pm 1$ subcomponents, i.e., transitions $S=0, M=0 \rightarrow S=1, M=0$ and $S=0, M=0 \rightarrow S=1, M= \pm 1$. Simulations using the Hamiltonian model (1) along this line indeed allow us to reproduce the energies of all observed transitions with good accuracy, yielding overall good fits to the experimental INS data. However, the intensity of feature I is grossly overestimated, and moreover, the obtained values for the single-ion anisotropy parameters $D_{\mathrm{Cr}}$ and $D_{\mathrm{Ni}}$ are one to two orders of magnitude too large (the reason will become clear below). Hence this interpretation is ruled out. Alternatively, peaks I and II can be interpreted as to be associated with the transitions from the $S=0$ ground state into two different excited $S=1$ spin multiplets, this ZFS is too small to be fully resolved in our experiments, i.e., smaller than approximately $0.1 \mathrm{meV}$ as suggested by the asymmetry of feature II seen in the IRIS data. The higher intensity at the lower energies in feature II then suggests that the ZFS parameter $D_{I I}$ of the second-excited $S=1$ multiplet giving rise to feature II is negative, such that its $M= \pm 1$ subcomponents are energetically lower lying than the $M=0$ subcomponent. Our analysis will confirm this picture.

The simulation of the INS results allowed a good discrimination between the two AFM exchange coupling constants $J_{\mathrm{CrNi}}$ and $J_{\mathrm{CrCr}}$. From simulations performed over a wide range of parameters it was found that the transitions III, IV, $\mathrm{V}$, and VI at high-energy transfer are very sensitive to $J_{\mathrm{CrCr}}$, with a best fit value of $J_{\mathrm{CrCr}}=1.32(5) \mathrm{meV}$, but exhibit a significantly weaker dependence on $J_{\mathrm{CrNi}}$ (see Sec. V B). Of the observed transitions the low-lying excitations I and II exhibit the strongest dependence on $J_{\mathrm{CrNi}}$. A best fit value of $J_{\mathrm{CrNi}}=3.22(25) \mathrm{meV}$ is found.

The experimental INS spectra can be simulated with the correct peak positions in energy and $Q$ with the HeisenbergDirac-van Vleck part of Eq. (1). However, in such a pure exchange model, the intensity of in particular transition II is significantly too large as compared to the measured spectra, and the asymmetry in this feature is not accounted for. Inclusion of single-ion anisotropy terms to the isotropic spin Hamiltonian improves significantly the agreement of the simulated intensities and shape of feature II with the measured INS spectra. Strain effects in the exchange constants, due to, e.g., the presence of the Ni(II) ion, which is disordered on the nine possible metal sites, can be expected to be present and may explain the observed broadening of transitions I and II, preventing a clear observation of a ZFS in the high-resolution IRIS data. Strain effects could, in principle, also be responsible for the asymmetry in feature II, however it can well be reproduced by ZFS and the shape of feature II is hence attributed to ZFS.

Several parameter sets for $D_{\mathrm{Cr}}$ and $D_{\mathrm{Ni}}$ were identified for which model (1) reproduces the experimental transitions energies well, but significant discrepancies in the intensities and/or unreasonable parameter values were observed. For instance, $D_{\mathrm{Cr}}=D_{\mathrm{Ni}}=0.25 \mathrm{meV}$ yield very good fits to the data, except that the intensity of transition I is overestimated by one order of magnitude. In previous INS and other experiments, the values $D_{\mathrm{Cr}}=-0.036 \mathrm{meV}$ and $D_{\mathrm{Ni}}=-0.43 \mathrm{meV}$ were reliably determined for the similar compound $\mathrm{Cr}_{7} \mathrm{Ni},{ }^{47}$ which suggests using them as approximations for the single-ion anisotropy in $\mathrm{Cr}_{8} \mathrm{Ni}$. Indeed, these values for $D_{\mathrm{Cr}}$ and $D_{\mathrm{Ni}}$, though the intensity of transition II is somewhat overestimated, give very good results. Also the splitting of approximately $0.1 \mathrm{meV}$ of feature II is very well reproduced, but the shape or the intensity pattern of the two underlying lines is not (inset to Fig. 6), since in this model, the ZFS parameter $D_{I I}$ of the involved $S=1$ multiplet is positive. From a magnetochemical point of view there can be little doubt that the value of $D_{\mathrm{Cr}}$ should be similar to those found in similar compounds, such as $\mathrm{Cr}_{7} \mathrm{Ni}$ or $\mathrm{Cr}_{8} \mathrm{Zn}^{25,47}$ and $D_{\mathrm{Cr}}=-0.036 \mathrm{meV}$ is a reliable estimate. However, in $\mathrm{Cr}_{8} \mathrm{Ni}$, the bridging paths involving the $\mathrm{Ni}$ ion are significantly different to those in $\mathrm{Cr}_{7} \mathrm{Ni}$, as evidenced also by the considerably different exchange constants $J_{\mathrm{CrNi}}$ in these two compounds, which suggests that $D_{\mathrm{Ni}}$ could be very different. Indeed, $D_{\mathrm{Ni}}=+1.25 \mathrm{meV}$ give equally good simulation results as before at the higher energies (for the 


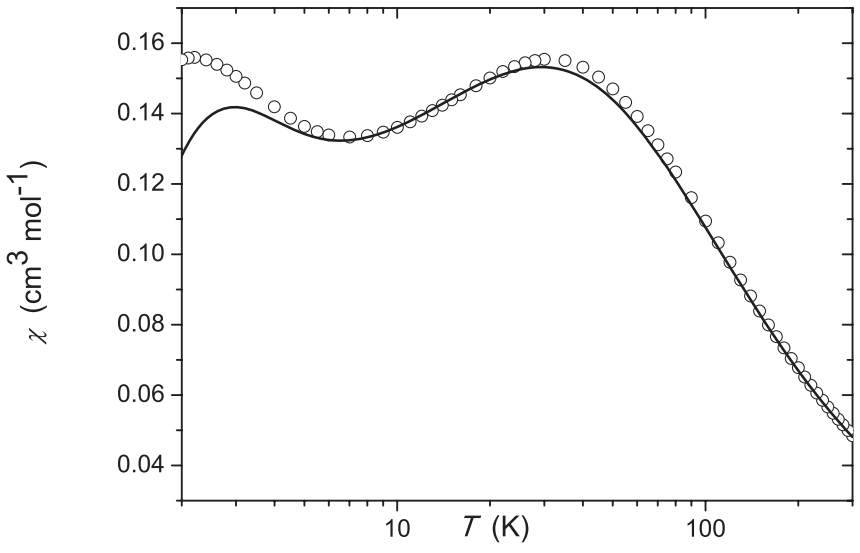

FIG. 7. The molecular susceptibility of $\mathrm{Cr}_{8} \mathrm{Ni}$ (black circles) at $1 \mathrm{KOe}$, measured in a temperature range of 2 to $300 \mathrm{~K}$. The black solid line shows theoretical calculated results based on Eq. (1) using the exchange constants determined from the INS experiments, $J_{\mathrm{CrCr}}=$ $1.32 \mathrm{meV}, J_{\mathrm{CrNi}}=3.22 \mathrm{meV}$, and Zeeman terms with $g_{\mathrm{Cr}}=1.98$ and $g_{\mathrm{Ni}}=2.2$.

IN5 data the simulations are essentially indistinguishable for $D_{\mathrm{Ni}}=-0.43$ and $+1.25 \mathrm{meV}$ ), but reproduces now the intensity pattern of feature II very well, since the parameter $D_{\text {II }}$ is obtained as negative. The single-ion anisotropy of $\mathrm{Ni}$ (II) ions can be large, and values of up to several meV were reported. ${ }^{48}$ We hence favor the parameters $D_{\mathrm{Cr}}=-0.036 \mathrm{meV}$ and $D_{\mathrm{Ni}}=+1.25 \mathrm{meV}$.

Simulated INS spectra using the determined parameters are compared to experiment in Fig. 5. A constant background was added to the calculated curves for each setting, and the elastic line was represented by a single gaussian. The satisfying good overall agreement is obvious. For comparison, without ZFS included in the model (1), the calculated intensity of transition II would be further enhanced by $20 \%$. At high-energy transfers, where the experimental nonmagnetic intensity is large relative to the magnetic transitions, a background was not added to the simulations to avoid potential systematic error. Figure 4(b) shows the simulated $S(Q, \omega)$ plot for the neutron scattering probed at $2.0 \mathrm{~K}$ with the $4.0 \AA$ setting. The simulations reproduce nicely the $Q$ dependence and energy transfer of the measured $S(Q, \omega)$ in Fig. 4(a). The intensity of peak II is too large as compared to the experiment by the reasons discussed for the integrated intensity spectra in the above.

In previous bulk magnetization studies, sizable (antisymmetric) Dzyaloshinski-Moriya (DM) interactions between the $\mathrm{Cr}$ and $\mathrm{Ni}$ ions were found to be necessary to reproduce the widths of magnetization steps and specific heat capacity at the magnetic field-induced level crossings. ${ }^{25}$ As regards the INS spectra, the inclusion of such DM interactions in the Hamiltonian model did not result in significant improvements.

INS spectra offer much more detailed information on the exchange couplings between the $\mathrm{Cr}$ and $\mathrm{Ni}$ sites than bulk measurements. To check for consistency, however, Fig. 7 shows the measured magnetic susceptibility and the theoretical curve calculated from the exchange parameters determined by INS and typical single-ion $g$ values for octahedral $\mathrm{Cr}$ (III) and $\mathrm{Ni}$ (II) ions. The susceptibility goes through a rounded maximum at about $25 \mathrm{~K}$. Homometallic chromium systems, which use the same bridging ligands as in $\mathrm{Cr}_{8} \mathrm{Ni}$, e.g., the $\mathrm{AFM}$ wheel $\mathrm{Cr}_{8}$ and the broken AFM wheel $\mathrm{Cr}_{8} \mathrm{Cd},{ }^{26,49}$ show similar temperature dependencies of the susceptibility in this temperature range, with a maximum $\left(\mathrm{Cr}_{8}\right)$ or inflection $\left(\mathrm{Cr}_{8} \mathrm{Cd}\right)$ at approximately $30 \mathrm{~K} . \mathrm{Cr}_{8} \mathrm{Ni}$ shows a second maxima at $2 \mathrm{~K}$, which reflects the presence of a very low-lying excited spin multiplet above the spin-zero ground state (transition I at $0.34 \mathrm{meV}$ ) with a significant gap to the next higher-lying multiplets (transition II at $0.98 \mathrm{meV}$ ), similar to the situation observed in the horseshoe molecule $\mathrm{Cr}_{6} \cdot{ }^{50}$ At low temperatures, the calculated susceptibility curve deviates slightly from the measured data, which could indicate weak terms in the spin Hamiltonian not present in Eq. (1). It can be concluded that the INS results identify the lowest-lying excited states correctly, and that the simplified model Hamiltonian (1) captures the main effects.

\section{DISCUSSION}

\section{A. Characterization and physical origin of the small ZFS}

The INS data confirm that in $\mathrm{Cr}_{8} \mathrm{Ni}$ the $J_{\mathrm{CrNi}}$ exchange is much stronger than $J_{\mathrm{CrCr}}$, with a ratio of $J_{\mathrm{CrNi}} / J_{\mathrm{CrCr}}=$ $2.44(21)$. The $J_{\mathrm{CrCr}}$ exchange constant for $\mathrm{Cr}_{8} \mathrm{Ni}$ is essentially identical to the $\mathrm{Cr}-\mathrm{Cr}$ coupling in the $\mathrm{Cr}_{8} \mathrm{Zn}$ compound with a similar geometry of the bridging ligands, for which $J_{\mathrm{CrCr}}=1.32 \mathrm{meV}$ was deduced from INS experiments. ${ }^{51}$ $J_{\mathrm{CrCr}}$ is, however, $10 \%$ smaller than in the eight-membered $\mathrm{Cr}$ (III) AFM wheels, such as $\mathrm{Cr}_{8},{ }^{52}$ or the heterometallic series of $\mathrm{Cr}_{7} \mathrm{M}$ wheels, were $\mathrm{M}$ is one of several possible $2+$ metal ions. ${ }^{47}$ The inclusion of an extra metal ion opens up the wheel and alters the geometry of the bridging ligands, which is proposed to be responsible for the different $\mathrm{Cr}-\mathrm{Cr}$ exchange constants. The inferred $J_{\mathrm{CrNi}}$ exchange constant for $\mathrm{Cr}_{8} \mathrm{Ni}$ is significantly smaller than that determined from the initial fits to the magnetic susceptibility data reported in Ref. $24\left(J_{\mathrm{CrNi}}=6.04 \mathrm{meV}\right)$ and the recent calculations based on magnetization data in Ref. $25\left(J_{\mathrm{CrNi}}=7.33 \mathrm{meV}\right)$. In these works, it was found that $J_{\mathrm{CrNi}}$ is mostly sensitive to low-temperature susceptibility. INS allowed us to probe these lowest lying states directly and to fix $J_{\mathrm{CrNi}}$. Despite the significantly smaller $J_{\mathrm{CrNi}} / J_{\mathrm{CrCr}}$ ratio obtained in this work as compared to the previous estimates, it is still large enough to stabilize the $S=0$ ground state in $\mathrm{Cr}_{8} \mathrm{Ni}$ (vide infra).

The simulated INS spectra were improved by the inclusion of single-ion anisotropy in the spin Hamiltonian. Calculations revealed that the single-ion anisotropy terms cause a larger ZFS of the second $S=1$ excited state than for the other transitions, which explains the decrease of the relative intensity of transition II as compared to a pure exchange model. The high-resolution INS measurements hinted towards an asymmetry in the scattering intensities of feature II, which could be attributed to the ZFS of the second excited $S=1$ state involved in feature II. The previous bulk measurements on $\mathrm{Cr}_{8} \mathrm{Ni}$ have not given evidence for a $\mathrm{ZFS}$ or single-ion anisotropy; the present INS study provides the experimental evidence for single-ion anisotropy in $\mathrm{Cr}_{8} \mathrm{Ni}$.

Despite single-ion anisotropy parameters of significant strength $\left(D_{\mathrm{Cr}}=-0.036 \mathrm{meV}, D_{\mathrm{Ni}}=+1.25 \mathrm{meV}\right)$, the calculated ZFS of the observed spin multiplets is rather small and was too small to be fully resolved in the INS experiments, 
TABLE I. Projections coefficients $d_{i}$ for the two lowest excited $S=1$ spin multiplets in $\mathrm{Cr}_{8} \mathrm{Ni}$. The numbering of the sites starts from the $\mathrm{Ni}$ ion $(i=0)$.

\begin{tabular}{lrrrrr}
\hline \hline multiplet & $\mathrm{Ni}$ & $\mathrm{Cr}_{1}, \mathrm{Cr}_{8}$ & $\mathrm{Cr}_{2}, \mathrm{Cr}_{7}$ & $\mathrm{Cr}_{3}, \mathrm{Cr}_{6}$ & $\mathrm{Cr}_{4}, \mathrm{Cr}_{5}$ \\
\hline I & 0.0784 & 0.4197 & 0.0683 & -0.1094 & -0.2503 \\
II & -0.1387 & -0.6439 & -0.4426 & 0.0572 & 0.2366 \\
\hline \hline
\end{tabular}

which is in striking contrast to the situation in the even AFM wheels, where single-ion anisotropy leads to a ZFS of the first excited $S=1$ level of several $0.1 \mathrm{meV}$. Within the strong-exchange limit, which is well obeyed in $\mathrm{Cr}_{8} \mathrm{Ni}$, the uniaxial ZFS parameter of a spin multiplet is given by the formula $D=\sum_{i} d_{i} D_{i},{ }^{53}$ where $d_{i}$ is a projection coefficient for the site $i$ and $D_{i}$ assumes either the value of $D_{\mathrm{Cr}}$ or $D_{\mathrm{Ni}}$ depending on the site. The projection coefficients depend also on the considered spin multiplet. For $\mathrm{Cr}_{8} \mathrm{Ni}$, the calculated projection coefficients for the first and second excited $S=1$ multiplets, which give rise to the INS features I and II, are listed in Table I. They were calculated exactly to within numerical precision using the wave functions obtained by full exact diagonalization and the formulas in Ref. 53. They yield the relations:

multiplet I: $D_{I}=0.2568 D_{\mathrm{Cr}}+0.0784 D_{\mathrm{Ni}}$,

multiplet II: $D_{I I}=-1.5855 D_{\mathrm{Cr}}-0.1387 D_{\mathrm{Ni}}$.

It is noted that for $D_{\mathrm{Ni}}=-0.43$ and $+1.25 \mathrm{meV}$ one obtains $D_{\mathrm{II}}=+0.117 \mathrm{meV}$ and $-0.117 \mathrm{meV}$, such that both parameters closely reproduce the observed splitting of feature II but with opposite sign, which directly explains the different shapes of the simulated INS curve (insert to Fig. 6). For spin multiplet I, $D_{\mathrm{I}}=-0.107 \mathrm{meV}$. This calculation also explains why the strong-exchange limit is well realized in $\mathrm{Cr}_{8} \mathrm{Ni}$. As demonstrated before for AFM wheels and other systems, ${ }^{13}$ what matters are not the actual values of the single-ion anisotropies but their combined effect on the exchange-split energy spectrum, which despite a large value of $D_{\mathrm{Ni}}$ in relation to the exchange constants is relatively small. That is, one should compare the ZFS of $\sim 0.1 \mathrm{meV}$ to the exchange splitting of $\sim 0.4 \mathrm{meV}$ (for spin multiplet I). For higher-lying spin multiplets the effects of ZFS become even weaker.

The projection coefficient argument also shows that if peaks I and II would correspond to transitions from the $S=0$ ground state into the anisotropy-split first excited $S=1$ multiplet, or their energy difference of $0.56 \mathrm{meV}$ to the ZFS of the first excited $S=1$ multiplet, as it was discussed as a possible interpretation of the data in the above, the single-ion anisotropy constants would have to be about $0.52 \mathrm{meV} / 0.1 \mathrm{meV}$ or five times larger than currently inferred, which clearly rules out this scenario.

Most interestingly, the projection coefficients $d_{i}$ reflect the internal spin structure in the associated spin multiplet. For a bipartite lattice, one expects them to be of the same sign for each lattice site $i$, since the spins on the two AFM sublattices are generically collinear. Thus the single-ion anisotropies $D_{i}$ add up constructively to give a comparatively large cluster ZFS (if the single-ion anisotropy tensors are dominantly "parallel" to each other, where "parallel" is to be understood in the obvious sense). However, for the odd wheels in the regime of strong frustration (see Sec. V C) one expects a curled spiral- like spin structure, as it had indeed been confirmed in previous works on $\mathrm{Cr}_{8} \mathrm{Ni}^{24,26}$ Hence, the projection coefficients are expected to change their sign along the wheel, exactly as demonstrated in Table I. For instance, for an octanuclear $\mathrm{Cr}$ (III) AFM wheel, the ZFS of the first-excited $S=1$ multiplet would be given as $D_{\mathrm{I}}=-4.977 D_{\mathrm{Cr}}$, i.e., would be boosted up by a factor of 5. In contrast, in $\mathrm{Cr}_{8} \mathrm{Ni}$ the contribution of the eight $\mathrm{Cr}$ (III) ions adds up to only $0.2568 D_{\mathrm{Cr}}$ in the first excited $S=1$ level, and inspection of Table I immediately evidences that this about 20 times smaller ZFS is intrinsically related to the change in sign of the $d_{i}$, and not to the $d_{i}$ being very small in magnitude. Importantly, the exchange splittings are not as much reduced since these are determined by the spin-spin correlations $\left\langle\hat{\mathbf{s}}_{i} \cdot \hat{\mathbf{s}}_{i+1}\right\rangle$, or in simple terms by the mutual angles between neighboring spins, which are still close to antiparallel in Cr8Ni (see, e.g., the discussion in Ref. 25). Therefore the relatively small cluster anisotropy, as compared to bipartite AFM systems, reflects the spiral-like or Möbius-strip-like internal spin structure of the AFM states in $\mathrm{Cr}_{8} \mathrm{Ni}$.

\section{B. Energy spectrum and comparison to even AFM wheels and related bipartite lattices}

Even-membered AFM wheels such as $\mathrm{CsFe}_{8},{ }^{12} \mathrm{Fe}_{10},{ }^{11}$ $\mathrm{Cr}_{8},{ }^{10}$ or $\mathrm{Cr}_{7} \mathrm{M},{ }^{47}$ as well as bipartite AFM [3 $\left.\times 3\right]$ grid molecules $^{54}$ have all been shown by INS measurement to follow a general spectral trend when energy is plotted as function of the total spin $S$ or $S(S+1)$. In such plots, the structure of the energy levels in these bipartite AFM systems can be divided into three distinct regions. ${ }^{55-57}$ The lowest-lying states for each $S$ are denoted as the $L$ band (but are also known as the tower of states $^{8}$ or quasidegenerate joint states ${ }^{57}$ ). The states with slightly higher energies than the $L$ band states are denoted as the $E$ band according to the selection rule, that large INS transition intensities are observed only between the $L$ - and $E$-band states (when corresponding to allowed $\Delta S=0, \pm 1$ selection rules). The remaining states in the energy spectrum, the so-called quasicontinuum, show negligible transition intensity. The spectral distribution of transition intensities is connected to the spin-spin pair correlation functions that determine the oscillator strengths of spin transitions. ${ }^{55}$ Furthermore the aforementioned $L$-band states closely follow $E(S) \propto S(S+1) .^{55,58}$

Figure 8 presents the numerically calculated low-lying energy levels in $\mathrm{Cr}_{8} \mathrm{Ni}$ as determined from the INS experiments as function of total spin $S$. For clarity, the ZFS within the spin multiplets due to the single-ion anisotropy is excluded from the figure. The experimentally observed transitions are indicated and labeled as in the previous sections. The structure of the energy levels differs significantly from the trends observed in the bipartite AFM wheels and grids, and the dependence of the energies of the lowest-lying spin multiplet in each $S$ sector is not well approximated by $S(S+1)$. Furthermore, clearly separated $L$ and $E$ bands can not be defined. The transitions I, II, and III emanating from the $S=0$ ground state exhibit the strongest scattering intensities, but the intensities for the higher-lying transitions IV to VI are significant, and a separation of the higher-lying energies into $E$ band and quasicontinuum as for the bipartite AFM wheels is not justified. The $Q$ dependence of the INS intensity reflects the 


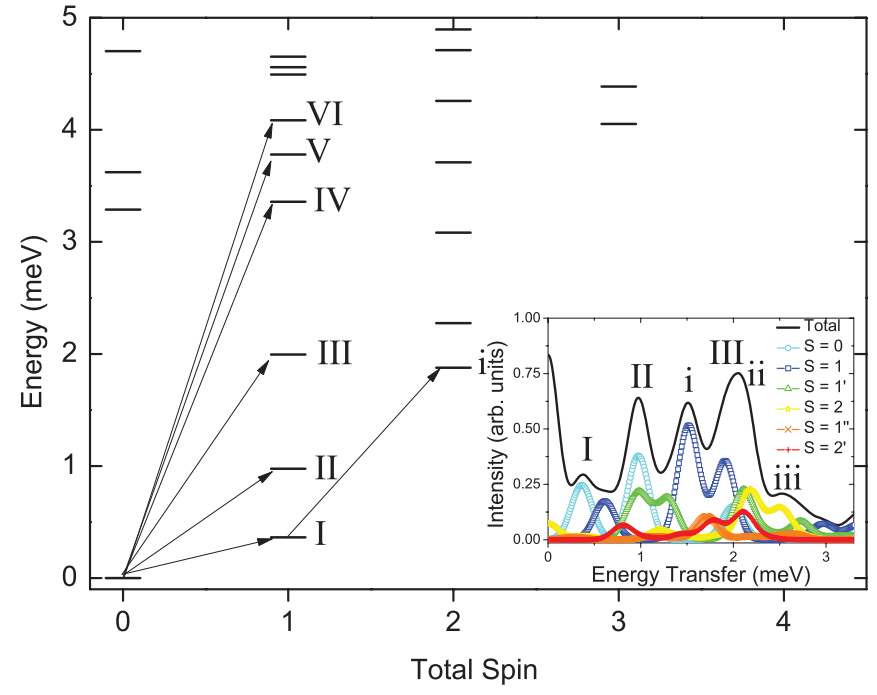

FIG. 8. (Color online) Low-lying energy spectrum for $\mathrm{Cr}_{8} \mathrm{Ni}$ as function of total spin $S$, calculated with the Heisenberg-Dirac-van Vleck part of Eq. (1) using $J_{\mathrm{CrCr}}=1.32 \mathrm{meV}$ and $J_{\mathrm{CrNi}}=3.22 \mathrm{meV}$. Labeled arrows indicate observed INS transitions. The observed hot peaks ii and iii have significant contributions from several transitions and are not indicated in the figure. The inset shows the individual contributions of the transition intensities coming from each of the low-lying spin states at $14.5 \mathrm{~K}$.

structure of the wave functions involved in the transitions. For $\mathrm{Cr}_{8} \mathrm{Ni}$, the $Q$ dependencies of transitions I, II, and III are similar, which is in marked contrast to the bipartite AFM wheels, where the $L$ - and $E$-band excitations were demonstrated both experimentally and theoretically to display fundamentally different $Q$ dependencies due to the different physical nature of these excitations. ${ }^{10}$ The breakdown of the generic $L$ - and $E$-band picture is due to the nonbipartite nature of an odd-membered AFM wheel, and the $Q$ dependencies observed in $\mathrm{Cr}_{8} \mathrm{Ni}$ provide the most clear-cut experimental signature for that.

Presumably general results for some properties of the lowlying excited states of AFM wheels, including odd-membered wheels, exists for homometallic systems. ${ }^{30}$ However, these results are inapplicable to $\mathrm{Cr}_{8} \mathrm{Ni}$ due to the presence of the spin-1 $\mathrm{Ni}$ (II) ion and the difference in the $J_{\mathrm{NiCr}}$ and $J_{\mathrm{CrCr}}$ exchange interactions.

\section{Effective models}

As discussed in the previous section, the $L$ - and $E$-band concept, which builds on a bipartite AFM sublattice structure fails, as expected, ${ }^{55}$ for the odd-membered $A F M$ wheel $\mathrm{Cr}_{8} \mathrm{Ni}$, and the question emerges if alternative simplified effective spin models can be obtained. In this context, it is of interest to discuss the dependence of the energy spectrum on the ratio $J^{\prime}=J_{\mathrm{CrNi}} / J_{\mathrm{CrCr}}$ in more detail. Only the exchange part in Eq. (1) is considered in the following, and energies are given in units of $J_{\mathrm{CrCr}}$ and measured with respect to the ground-state energy. The energy spectrum versus $J^{\prime}$ is plotted for a relevant range in Fig. 9. Similar results were presented before. ${ }^{27}$

As regards the AFM region $J^{\prime}>0$, the two regimes $J^{\prime} \ll 1$ (weak $J_{\mathrm{CrNi}}$ ) and $J^{\prime} \gg 1$ (strong $J_{\mathrm{CrNi}}$ ) can clearly be
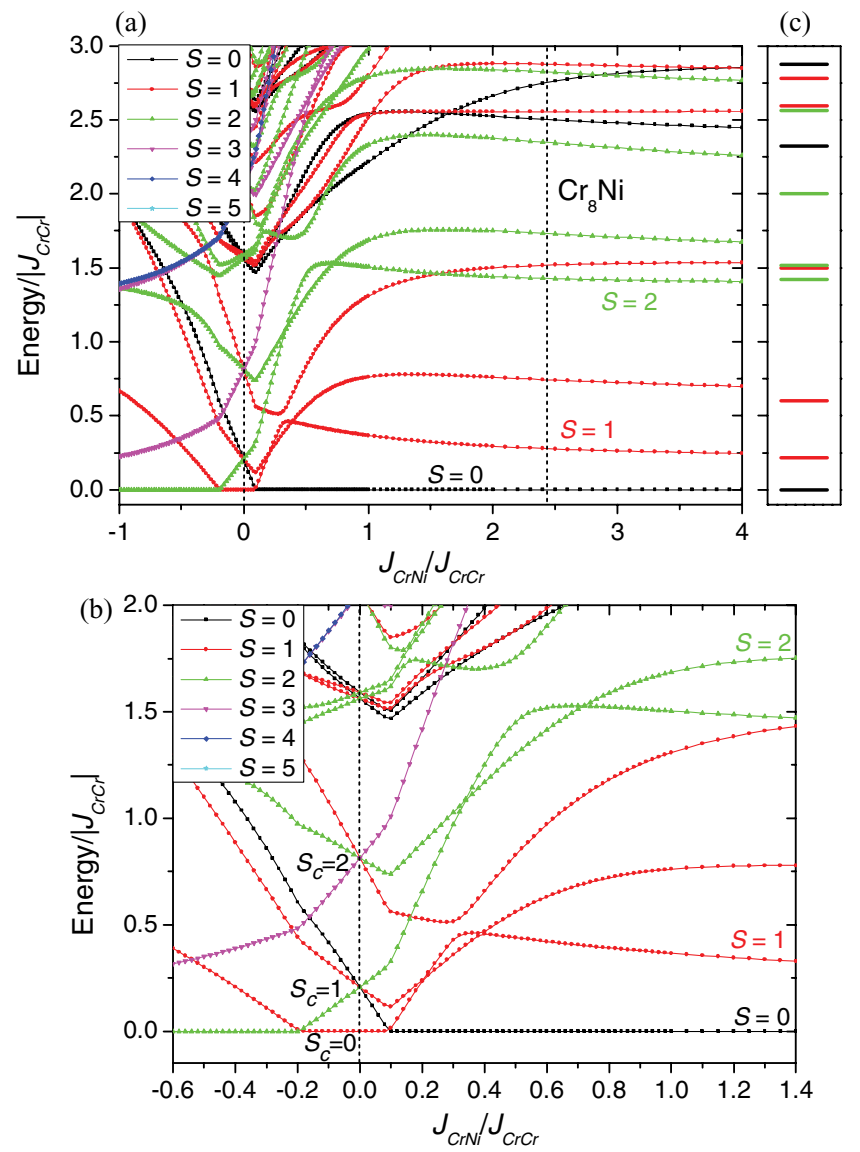

FIG. 9. (Color online) (a) and (b) show with different detail the low-lying energy spectrum for $\mathrm{Cr}_{8} \mathrm{Ni}$ as a function of the ratio $J^{\prime}=$ $J_{\mathrm{CrNi}} / J_{\mathrm{CrCr}}$, calculated with the Heisenberg-Dirac-van Vleck part of Eq. (1). (c) The low-lying energies as calculated with the effective seven-membered AFM wheel model discussed in the text. Energies are given in units of $J_{\mathrm{CrCr}}$ and with respect to the ground state.

identified, for instance, by the bending of the energy levels in the crossover region. Furthermore, a ground-state level crossing at $J^{\prime *}=0.09389$, where the ground state changes from $S=1$ to $S=0$ can be observed. Interestingly, and importantly, it occurs at a value of $J^{\prime}$ at which the energy levels do not yet display the strong bending characterizing the crossover from $J^{\prime} \ll 1$ to $J^{\prime} \gg 1$. The ground-state level crossing does hence not qualify as a criterion for distinguishing these regimes. The key features in the evolution of the energy spectrum with $J^{\prime}$ are generic for odd AFM wheels with one inequivalent spin center $\left(\hat{\mathbf{s}}_{0}\right)$, and the observed trend for $\mathrm{Cr}_{8} \mathrm{Ni}$ will be discussed in a manner which permits obvious extension to more general cases.

For not too large $J^{\prime}$, the spin states of the cluster may be constructed by coupling the inequivalent spin $\hat{\mathbf{s}}_{0}$ with the spin states of the remaining even-membered "chain" of eight spins of length $s$. The states on the chain shall be classified by the spin quantum number $S_{c}$ and the eigenstates of the respective Hamiltonian [first term in Eq. (1)] can be written as $\left|\alpha S_{c} M_{c}\right\rangle$, where $\alpha$ denotes any quantum numbers required for unambiguous classification of the states. The ground state of the chain belongs to $S_{c}=0$ followed by $S_{c}=1$ and 2 excited states (and further higher-lying spin states not considered 
here). The spin states of the total cluster can then be constructed from the product states $\left|s_{0} m_{0}\right\rangle\left|\alpha S_{c} M_{c}\right\rangle$, yielding coupled states $\left|\alpha s_{0} S_{c} S M\right\rangle$ with $S=\left|s_{0}-S_{c}\right|, \ldots, s_{0}+S_{c}$. For very small $J^{\prime}$, the $S_{c}=0$ ground state of the chain gives rise to a $S=s_{0}$ ground state of the cluster, or $S=1$ for $\mathrm{Cr}_{8} \mathrm{Ni}$. The first excited $S_{c}=1$ chain state generates cluster states with $S=0,1,2$ and the next excited $S_{c}=2$ chain state cluster states with $S=1,2,3$, as confirmed in Fig. 9 .

With increasing $J^{\prime}$, higher-lying states come down, and the ground state undergoes a sequences of changes from $S=s_{0}$ to $S=s_{0}-1, S=s_{0}-2$, and so on. This continues until $S$ assumes its lowest possible value of $S=1 / 2$ or 0 depending on the number of magnetic electrons in the system. Up to this point, the wave functions may reasonably well be approximated by the spin-coupled states $\left|\alpha s_{0} S_{c} S M\right\rangle$, which corresponds to a first-order perturbation treatment of the exchange couplings $J_{\mathrm{CrNi}}$ [or the second term in Eq. (1)]. For $\mathrm{Cr}_{8} \mathrm{Ni}$, this mechanism results in only one ground-state level crossing (see Fig. 9). In first order, the energy shifts due to the perturbation $\left(\hat{\mathbf{s}}_{1}+\hat{\mathbf{s}}_{8}\right) \cdot \hat{\mathbf{s}}_{0}$ can be calculated to $2 c_{18} \hat{\mathbf{S}}_{c} \cdot \hat{\mathbf{s}}_{0}=c_{18}\left(S^{2}-S_{c}^{2}-s_{0}^{2}\right)$, where $c_{18}$ is the projection coefficient of the spin $\hat{\mathbf{s}}_{1}$ or $\hat{\mathbf{S}}_{8}$ on the chain spin $\hat{\mathbf{S}}_{c}$. For the first excited chain state in $\mathrm{Cr}_{8} \mathrm{Ni}$, one calculates numerically $c_{18}=0.54213$ and an energy gap of 0.20733 . This results in the estimate $J^{\prime *}=0.09561$ for the occurrence of the ground-state level crossing, which closely agrees with the exact value of $J^{\prime *}=0.09389$. The accuracy of the first-order treatment in the regime $J^{\prime} \ll 1$ reflects the weak correlations between the inequivalent spin $\hat{\mathbf{s}}_{0}$ and the spins on the chain. Physically, this situation is not of most interest despite ground-state level crossings occurring.

Beyond that point, or the last level crossing, the system can, however, not be described as an even-membered chain plus inequivalent spin anymore, corresponding to the buildup of strong quantum correlations (entanglement) between these two subsystems or spin frustration indeed. For $J^{\prime} \gg$ 1 , the generic properties of the low-lying energy spectrum become essentially independent on the value of $J^{\prime}$ or $J_{\mathrm{CrNi}}$, as is also evident from Fig. 9. In this regime, spin frustration is maximally developed. This regime is obviously of most interest and is realized in $\mathrm{Cr}_{8} \mathrm{Ni}$. It is mentioned in passing that the near independence of the energy spectrum on $J_{\mathrm{CrNi}}$ in this regime explains the earlier finding in Sec. IV that $J_{\mathrm{CrCr}}$ could be determined from the experimental data with better accuracy than $J_{\mathrm{CrNi}}$ and may explain the significantly different values for $J_{\mathrm{CrNi}}$ obtained by the different techniques as discussed in Sec. V A. In this regime, as noted before by several authors, ${ }^{24,25,27,28}$ the $\mathrm{Ni}$ (II) spin $\hat{\mathbf{s}}_{0}$ is essentially antiparallel to the neighboring spins $\mathrm{Cr}$ (III) spins $\hat{\mathbf{s}}_{1}$ and $\hat{\mathbf{s}}_{8}$ and the ground state has been pictured as a VBS. ${ }^{28}$

In this situation of large $J^{\prime} \gg 1$, another simplified effective model can be derived by integrating out the excitations on the "trimer" formed by the inequivalent spin $\hat{\mathbf{s}}_{0}$ and its two neighboring spins, $\hat{\mathbf{s}}_{1}$ and $\hat{\mathbf{s}}_{8}$, or the second term in Eq. (1). The eigenfunctions of this trimer may be written as $\left|s_{1} s_{8} S_{18} s_{0} S_{t} M_{t}\right\rangle$ in an obvious notation, and their energies are $E_{t}=\frac{1}{2} J_{\mathrm{CrNi}}\left[S_{t}\left(S_{t}+1\right)-S_{18}\left(S_{18}+1\right)\right]$. The wave functions on the remaining even-membered "reduced chain" of the six spins $\hat{\mathbf{s}}_{2}$ to $\hat{\mathbf{s}}_{7}$ are written as $|n\rangle$, where $n$ denotes any set of suitable quantum numbers, and basis functions for the total cluster could be constructed as the product states $|n\rangle\left|s_{1} s_{8} S_{18} s_{0} S_{t} M_{t}\right\rangle$. Then, in first order, the exchange model (1) reduces to

$$
\hat{H}_{1}=J_{\mathrm{CrCr}}\left[\sum_{i=2}^{6} \hat{\mathbf{s}}_{i} \cdot \hat{\mathbf{s}}_{i+1}+c_{18}^{t}\left(\hat{\mathbf{s}}_{2} \cdot \hat{\mathbf{S}}_{t}+\hat{\mathbf{S}}_{t} \cdot \hat{\mathbf{s}}_{7}\right)\right],
$$

where a trivial constant is omitted and where

$$
c_{18}^{t}=\frac{S_{t}\left(S_{t}+1\right)+S_{18}\left(S_{18}+1\right)-s_{0}\left(s_{0}+1\right)}{4 S_{t}\left(S_{t}+1\right)}
$$

is the projection coefficient of the spin $\hat{\mathbf{s}}_{1}$ or $\hat{\mathbf{s}}_{8}$ on the trimer spin $\hat{\mathbf{S}}_{t}{ }^{53}$ The lowest-lying set of states is obtained from the trimer ground state, which belongs to $S_{t}=\left|2 s-s_{0}\right|$.

Hence, for $J^{\prime} \gg 1$, the originally nine-membered odd wheel reduces to a seven-membered odd wheel with an inequivalent spin of length $s_{0}=S_{t}$ and nearest-neighbor couplings of strengths $c_{18}^{t} J_{\mathrm{CrCr}}$. For $\mathrm{Cr}_{8} \mathrm{Ni}, S_{t}=2$ and $c_{18}^{t}=$ $2 / 3$ holds, and the resulting low-lying energies of Eq. (2) are plotted in Fig. 9(c). The comparison with Fig. 9(a) reveals that the reduced odd chain model (2) does not perfectly describe the energy spectrum in $\mathrm{Cr}_{8} \mathrm{Ni}$, but the similarity is obvious, and could significantly be improved by a small renormalization of the exchange constants $J_{\mathrm{CrCr}}$ and $c_{18}^{t} J_{\mathrm{CrCr}}$ in Eq. (2) accounting for higher-order contributions. This effective model represents a significant simplification over the original model, as the Hilbert space in the case of $\mathrm{Cr}_{8} \mathrm{Ni}$ is reduced by an order of magnitude. However, it cannot further be reduced, at least not trivially, and, in particularly, not to a sublattice Hamiltonian as for the even-membered AFM wheels or the $\mathrm{Fe}_{30}$ Keplerate molecule. Hence the gain in insight it provides is limited, which reflects the lack of partitedness in odd wheels and emphasizes the distinctiveness of this class of molecular nanomagnets.

\section{CONCLUSION}

The inelastic magnetic neutron scattering profile of $\mathrm{Cr}_{8} \mathrm{Ni}$ revealed the energy and momentum transfer dependence of the low-lying spin excitations in detail, enabling an accurate characterization of its magnetic properties. A microscopic spin Hamiltonian was used to simulate the INS spectra giving isotropic exchange coupling parameters $J_{\mathrm{CrCr}}=1.32 \mathrm{meV}$ and $J_{\mathrm{CrNi}}=3.22 \mathrm{meV}$. A careful analysis of the relative INS transition intensities revealed clear indication that single-ion anisotropy is present but weak within $\mathrm{Cr}_{8} \mathrm{Ni}$. The determined $J_{\mathrm{CrNi}}$ exchange is $44 \%$ smaller than that previously deduced from the simulation of bulk susceptibility data. ${ }^{25}$ The $J_{\mathrm{CrNi}}$ exchange is strong enough to stabilize a singlet ground state and, moreover, to place $\mathrm{Cr}_{8} \mathrm{Ni}$ in the regime in which quantum correlations (entanglement) or spin frustration are strongest and in which the singlet ground state can be discussed in the framework of the proposed valence bond solid model. ${ }^{28}$ It will be of interest to see if this type of model could be applied to other odd-membered heterometallic rings. The energy versus total spin spectral trend of the low-lying eigenstates was analyzed. It is concluded that the odd-membered wheel does not exhibit the spectral trends observed for bipartite AFM wheels and related systems. Finally, it was demonstrated that the relatively weak magnetic anisotropy or ZFS of the spin multiplets, in relation to the expected size bipartite AFM lattices, is directly connected to the curly, spiral-like intrinsic 
spin structure in $\mathrm{Cr}_{8} \mathrm{Ni}$, hence providing further experimental evidence for the spin frustration in this molecule.

\section{ACKNOWLEDGMENTS}

This research project has been supported by the European Commission under the 7th Framework Programme through the "Research Infrastructures" action of the "Capacities" Programme, Contract No. CP-CSA INFRA-2008-1.1.1 Number
226507-NMI3. We are grateful to the EC NoE MagMaNet, the EPSRC, and to the ILL for funding. We are appreciative to the the EPSRC National EPR Facility, ILL, PSI, and ISIS for the access to experimental facilities. R.E.P.W. is grateful to the Royal Society for a Wolfson Merit Award. M.L.B. thanks the Japan society for the promotion of science for a postdoctoral fellowship. S.P. thanks the Danish Natural Science Research Council for a Steno Fellowship (09-062877) and a Sapere Aude Fellowship (10-081659).
*Also at Department of Physics and Astronomy, University College London, Gower Street, London, WC1E 6BT, United Kingdom.

${ }^{1}$ J. Schnack, Dalton Trans. 39, 4677 (2010).

${ }^{2}$ O. Waldmann, Coord. Chem. Rev. 249, 2550 (2005).

${ }^{3}$ D. Gatteschi, R. Sessoli, and J. Villain, Molecular Nanomagnets (Oxford University Press, Oxford, 2006).

${ }^{4}$ M. Affronte, F. Troiani, A. Ghirri, S. Carretta, P. Santini, V. Corradini, R. Schuecker, C. Muryn, G. Timco, and R. E. P. Winpenny, Dalton Trans. 2810 (2006).

${ }^{5}$ E. del Barco, A. D. Kent, S. Hill, J. M. North, N. S. Dalal, E. M. Rumberger, D. N. Hendrickson, N. Chakov, and G. Christou, J. Low Temp. Phys. 140, 119 (2005).

${ }^{6}$ P. Kögerler, B. Tsukerblat, and A. Müller, Dalton Trans. 39, 21 (2010).

${ }^{7}$ P. W. Anderson, Phys. Rev. 86, 694 (1952).

${ }^{8}$ P. W. Anderson, Basic Notions of Condensed Matter Physics (Benjamin/Cummings, Menlo Park, California, 1984).

${ }^{9}$ E. Lieb and D. Mattis, J. Math. Phys. 3, 749 (1962).

${ }^{10}$ O.Waldmann, T. Guidi, S. Carretta, C. Mondelli, and A. L. Dearden, Phys. Rev. Lett. 91, 237202 (2003).

${ }^{11}$ P. Santini, S. Carretta, G. Amoretti, T. Guidi, R. Caciuffo, A. Caneschi, D. Rovai, Y. Qiu, and J. R. D. Copley, Phys. Rev. B 71, 184405 (2005).

${ }^{12}$ J. Dreiser, O. Waldmann, C. Dobe, G. Carver, S. T. Ochsenbein, A. Sieber, H.-U. Güdel, J. van Duijn, J. Taylor, and A. Podlesnyak, Phys. Rev. B 81, 024408 (2010).

${ }^{13}$ O. Waldmann, T. C. Stamatatos, G. Christou, H. U. Güdel, I. Sheikin, and H. Mutka, Phys. Rev. Lett. 102, 157202 (2009).

${ }^{14}$ S. Carretta, P. Santini, G. Amoretti, T. Guidi, J. R. D. Copley, Y. Qiu, R. Caciuffo, G. Timco, and R. E. P. Winpenny, Phys. Rev. Lett. 98, 167401 (2007).

${ }^{15}$ G. Chaboussant, R. Basler, A. Sieber, S. T. Ochsenbein, A. Desmedt, R. E. Lechner, M. T. F. Telling, P. Kögerler, A. Müller, and H.-U. Güdel, Europhys. Lett. 59, 291 (2002).

${ }^{16}$ G. Chaboussant, S. T. Ochsenbein, A. Sieber, H.-U. Güdel, H. Mutka, A. Müller, and B. Barbara, Europhys. Lett. 66, 423 (2004).

${ }^{17}$ Y. Furukawa, Y. Nishisaka, K. I. Kumagai, P. Kögerler, and F. Borsa, Phys. Rev. B 75, 220402 (2007).

${ }^{18}$ J. Yoon, L. M. Mirica, T. D. P. Stack, and E. I. Solomon, J. Am. Chem. Soc. 39, 395 (2004).

${ }^{19}$ H. T. Diep, Frustrated Spin Systems (World-Scientific, Menlo Park, 2005).

${ }^{20}$ I. Rousochatzakis, A. M. Läuchli, and F. Mila, Phys. Rev. B 77, 094420 (2008).
${ }^{21}$ J. Schulenburg, A. Honecker, J. Schnack, J. Richter, and H.-J. Schmidt, Phys. Rev. Lett. 88, 167207 (2002).

${ }^{22}$ H. C. Yao, J. J. Wang, Y. S. Ma, O. Waldmann, W. X. Du, Y. Song, Y.Z. Li, L. M. Zheng, S. Decurtins, and X. Q. Xin, Chem. Commun. 1745 (2006).

${ }^{23}$ N. Hoshino, M. Nakano, H. Nojiri, W. Wernsdorfer, and H. Oshio, J. Am. Chem. Soc. 131, 15100 (2009).

${ }^{24}$ O. Cador, D. Gatteschi, R. Sessoli, F. K. Larsen, J. Overgaard, A.-L. Barra, S. J. Teat, G. A. Timco, and R. E. P. Winpenny, Angew. Chem., Int. Ed. 43, 5196 (2004).

${ }^{25}$ Y. Furukawa, K. Kiuchi, K.-i. Kumagai, Y. Ajiro, Y. Narumi, M. Iwaki, K. Kindo, A. Bianchi, S. Carretta, P. Santini, F. Borsa, G. A. Timco, and R. E. P. Winpenny, Phys. Rev. B 79, 134416 (2009).

${ }^{26}$ Y. Furukawa, K. Kiuchi, K. I. Kumagai, Y. Ajiro, Y. Narumi, M. Iwaki, K. Kindo, A. Bianchi, S. Carretta, G. A. Timco, and R. E. P. Winpenny, Phys. Rev. B 78, 092402 (2008).

${ }^{27}$ O. Cador, D. Gatteschi, R. Sessoli, A.-L. Barra, G. A. Timco, and R. E. P. Winpenny, J. Magn. Magn. Mater. 290-291, 55 (2004).

${ }^{28}$ J. Almeida, M. A. Martin-Delgado, and G. Sierra, Phys. Rev. B 79, 115141 (2009).

${ }^{29}$ J. Schnack, M. Luban, and R. Modler, Europhys. Lett. 56, 863 (2001).

${ }^{30}$ K. Bärwinkel, H.-J. Schmidt, and J. Schnack, J. Magn. Magn. Mater. 220, 227 (2000).

${ }^{31}$ K. Bärwinkel, P. Hage, H.-J. Schmidt, and J. Schnack, Phys. Rev. B 68, 054422 (2003).

${ }^{32}$ S. Rachel, Europhys. Lett. 86, 37005 (2009).

${ }^{33}$ I. Affleck, T. Kennedy, E. H. Lieb, and H. Tasaki, Phys. Rev. Lett. 7, 799 (1987).

${ }^{34}$ L. Balents, Nature (London) 464, 199 (2010).

${ }^{35}$ S. DiMatteo, G. Jackeli, C. Lacroix, and N. B. Perkins, Phys. Rev. Lett. 93, 077208 (2004).

${ }^{36}$ M. E. Zhitomirsky and K. Ueda, Phys. Rev. B 54, 9007 (1996).

${ }^{37}$ G. A. Timco, A. S. Batsanov, F. K. Larsen, C. A. Muryn, J. Overgaard, S. J. Teat, and R. E. P. Winpenny, Chem. Commun. 3649 (2005).

${ }^{38}$ O. Waldmann, Phys. Rev. B 68, 174406 (2003).

${ }^{39}$ O. Waldmann and H. U. Güdel, Phys. Rev. B 72, 094422 (2005).

${ }^{40}$ R. Caciuffo, T. Guidi, G. Amoretti, S. Carretta, E. Liviotti, P. Santini, C. Mondelli, G. Timco, C. A. Muryn, and R. E. P. Winpenny, Phys. Rev. B 71, 174407 (2005).

${ }^{41}$ O. Waldmann, Phys. Rev. B 61, 6138 (2000).

${ }^{42}$ E. R. Davidson, J. Comput. Phys. 17, 87 (1975). 
${ }^{43}$ S. Piligkos, E. Bill, D. Collison, E. J. L. McInnes, G. A. Timco, H. Weihe, R. E. P. Winpenny, and F. Neese, J. Am. Chem. Soc. 127, 760 (2007).

${ }^{44}$ S. Piligkos, H. Weihe, E. Bill, F. Neese, H. E. Mkami, G. M. Smith, D. Collison, G. Rajaraman, G. A. Timco, R. E. P. Winpenny, and E. J. L. McInnes, Chem. Eur. J. 15, 3152 (2009).

${ }^{45}$ J. van Slageren, S. Piligkos, and F. Neese, Dalton Trans. 39, 4999 (2010).

${ }^{46}$ M. L. Baker, S. Piligkos, A. Bianchi, S. Carretta, D. Collison, J. J. W. McDouall, E. J. L. McInnes, H. Mutka, G. A. Timco, F. Tuna, P. Vadivelu, H. Weihe, H.-U. Güdel, and R. E. P. Winpenny, Dalton Trans. 40, 8533 (2011).

${ }^{47}$ R. Caciuffo, T. Guidi, G. Amoretti, S. Carretta, E. Liviotti, P. Santini, C. Mondelli, G. Timco, C. A. Muryn, and R. E. P. Winpenny, Phys. Rev. B 71, 174407 (2005).

${ }^{48}$ J.-N. Rébilly, G. Charron, E. Riviére, R. Guillot, A.-L. Barra, M. Durán-Serrano, J. van Slageren, and T. Mallah, Chem. Eur. J. 14, 1169 (2008).

${ }^{49}$ J. van Slageren, R. Sessoli, D. Gatteschi, A. A. Smith, M. Helliwell, R. E. P. Winpenny, A. Cornia, A.-L. Barra, A. G. M. Jansen, E. Rentschler, and G. A. Timco, Chem. Eur. J. 8, 227 (2002).
${ }^{50}$ S. T. Ochsenbein, O. Waldmann, A. Sieber, G. Carver, R. Bircher, H. U. Güdel, R. S. G. Davies, G. A. Timco, R. E. P. Winpenny, H. Mutka, and F. Fernandez-Alonso, Europhys. Lett. 79, 17003 (2007).

${ }^{51}$ A. Bianchi, S. Carretta, P. Santini, G. Amoretti, T. Guidi, Y. Qiu, J. R. D. Copley, G. Timco, C. Muryn, and R. E. P. Winpenny, Phys. Rev. B 79, 144422 (2009).

${ }^{52}$ S. Carretta, J. van Slageren, T. Guidi, E. Liviotti, C. Mondelli, D. Rovai, A. Cornia, A. L. Dearden, F. Carsughi, M. Affronte, C. D. Frost, R. E. P. Winpenny, D. Gatteschi, G. Amoretti, and R. Caciuffo, Phys. Rev. B 67, 094405 (2003).

${ }^{53}$ A. Bencini and D. Gatteschi, EPR of Exchange Coupled Systems (Springer-Verlag, Berlin, 1990).

${ }^{54}$ T. Guidi, S. Carretta, P. Santini, E. Liviotti, N. Magnani, C. Mondelli, O. Waldmann, L. K. Thompson, L. Zhao, C. D. Frost, G. Amoretti, and R. Caciuffo, Phys. Rev. B 69, 104432 (2004).

${ }^{55}$ O. Waldmann, Phys. Rev. B 65, 024424 (2001).

${ }^{56} \mathrm{C}$. Lhuillier, e-print arXiv:cond-mat/0502464.

${ }^{57}$ B. Bernu, C. Lhuillier, and L. Pierre, Phys. Rev. Lett. 69, 2590 (1992).

${ }^{58}$ J. Schnack and M. Luban, Phys. Rev. B 63, 014418 (2000). 